\title{
Comparison of urinary odor-induced glomerular activation in the main olfactory bulb of aromatase knock-out and wild type female mice
}

\author{
K.L. Martel ${ }^{\mathrm{a}}$, M. Keller ${ }^{\mathrm{b}}$, Q. Douhard ${ }^{\mathrm{b}}$, J. Bakker ${ }^{\mathrm{b}}$, M.J. Baum ${ }^{\mathrm{a}, *}$ \\ a Department of Biology, Boston University, 5 Cummington St., Boston, MA 02215, USA \\ ${ }^{\mathrm{b}}$ Center for Cellular and Molecular Neurobiology, University of Liege, Ave. de l'hopital 1, B36, 4000 Liege, Belgium
}

Received 26 March 2007; received in revised form 1 May 2007; accepted 4 May 2007

\begin{abstract}
Previously [D.W. Wesson, M. Keller, Q. Douhard, M.J. Baum, J. Bakker, Enhanced urinary odor discrimination in female aromatase knockout mice, Horm. Behav. 49 (2006) 580-586] female aromatase knock out mice successfully learned to discriminate in a food-motivated go/no-go task between urinary volatiles from ovariectomized female mice treated with estradiol as opposed to estradiol plus progesterone whereas wild type females failed to learn this odor discrimination. We asked whether this behavioral difference is reflected in the ability of these two types of urinary volatiles to differentially stimulate Fos expression in juxtaglomerular cells (an index of glomerular activation) of the main olfactory bulb (MOB) in wild type versus ArKO female mice. Statistically significant differences in the profiles of MOB glomerular activation were seen in ovariectomized, estrogen-treated ArKO as well as WT female subjects following exposure to urinary volatiles from ovariectomized females given estradiol alone as opposed to estradiol plus progesterone. Therefore, previously observed differences between females of the two genotypes in their behavioral responses to these odors must reflect differential processing in more central segments of the olfactory pathway instead of in the MOB.

(C) 2007 Elsevier Ireland Ltd. All rights reserved.
\end{abstract}

Keywords: Estradiol; Progesterone; Pheromone; Sexual differentiation; Fos expression

Olfactory receptor neurons in the main olfactory epithelium (MOE) that express the same olfactory receptor gene extend axons that target the same 1-2 glomeruli in the ipsilateral main olfactory bulb (MOB) [10]. Recently [6] a class of 'trace amineassociated receptors' was identified in the MOE which respond to volatile amines emitted from mouse urine. A series of studies by Restrepo and co-workers $[13,12,7]$ showed that volatile urinary odors from male mice augmented Fos expression in juxtaglomerular cells of the MOB (an index of glomerular activation) of female conspecifics. More recently, Martel and Baum [9] used this same method to show that urinary volatiles from male versus estrous female mice activated overlapping, but distinguishable clusters of glomeruli located in the ventral portion of the MOB in both sexes. In another study [8] volatile constituents of mouse urine were shown to stimulate activity in MOB mitral cells. Wesson et al. [14] recently assessed the possible contribution of perinatal exposure to estradiol on the later ability of female mice to discriminate volatile urinary odors from

\footnotetext{
* Corresponding author. Tel.: +1 617353 3009; fax: +1 6173530996. E-mail address: baum@bu.edu (M.J. Baum).
}

conspecifics of different sexes and endocrine status. Detection of these social odorants plays a significant role in sexual, aggressive, and maternal behaviors in mice, therefore the possible role of perinatal estradiol exposure on the later ability of female mice to discriminate between urinary odors of conspecifics is of particular interest. Female mice with a null mutation of the Cyp-19 gene (ArKO mice), which encodes the estradiol synthesizing enzyme, aromatase, readily learned to discriminate urinary volatiles from ovariectomized females treated with estradiol (E) as opposed to estradiol sequenced with progesterone $(\mathrm{E}+\mathrm{P})$. Surprisingly, wild type (WT) females failed to acquire this discrimination in a food-motivated go/no-go task assessed using an automated liquid dilution 8-channel olfactometer [3]. Both WT and ArKO females successfully learned to discriminate between other types of volatile urinary odors, including those from gonadally intact male versus ovariectomized females primed with $\mathrm{E}+\mathrm{P}$ and those from gonadally intact versus castrated males [14]. In the present study, we asked whether the different capacities of WT and ArKO females to discriminate volatile urinary odors from ovariectomized females treated with $\mathrm{E}$ as opposed to $\mathrm{E}+\mathrm{P}$ (which is presumably a more difficult discrimination than the other above-mentioned pairs of odors) can 
be explained by a difference between females of these two genotypes in the ability of these two odors to activate distinguishable clusters of MOB glomeruli.

All experimental methods used were in accordance with the guidelines of the National Institutes of Health and were approved by the Boston University Institutional Animal Care and Use Committee as well as the Ethical Committee for Animal Use at the University of Liège, Belgium. Animals were individually housed under a $12 \mathrm{~h}$ light/dark photoperiod with food and water provided ad libitum. All odor exposure sessions were conducted during the light phase of the photoperiod. A total of 29 wild-type (WT) and homozygous-null mutant (ArKO) [5] mice were generated by breeding heterozygous males and females of the same strain $(C 57 B l / 6 j \times s v 129)$. Tail DNA was used to genotype the mice using PCR analysis as previously described [2]. From the time of weaning, all mice were housed in samesex groups of 3-4 females. At the age of 3 months, all subjects were ovariectomized under ketamine $(80 \mathrm{mg} / \mathrm{kg})$ and medetomidine $(1 \mathrm{mg} / \mathrm{kg})$ anesthesia, and received atipamezole at the end of surgery to decrease recovery time. Subjects were allowed 1 week to recover, and were given daily injections of estradiol benzoate (EB, $1 \mu \mathrm{g} /$ day) [14] dissolved in sesame oil for 7 days prior to odor exposure. This particular dose of EB is sufficient to activate sexual behavior in ovariectomized female mice and maximized ovariectomized subjects' ability to acquire odor discrimination tasks in the olfactometer.

Urine was obtained by holding the animal by the scruff of the neck over an odorless glass vial and gently applying pressure to the abdomen. Urine was collected from 10 ovariectomized female mice in each of two endocrine states: Estrous female $(\mathrm{E}+\mathrm{P})$ urine was obtained from mice that had been implanted with a silastic capsule containing crystallized estradiol (diluted $1: 1$ with cholesterol) 2 weeks prior to urine collection, and which received an s.c. injection of progesterone $(500 \mu \mathrm{g}$ dissolved in sesame oil) $3 \mathrm{~h}$ prior to collection. Estrogen-treated urine was obtained from the same stimulus females with silastic estradiol implants (implanted for at least 2 weeks prior to collection), but in the absence of progesterone priming. Urine from all 10 females of the same endocrine state was pooled, aliquotted, and stored at $-20^{\circ} \mathrm{C}$.

Subjects were individually housed in clean cages and food deprived 1 day before odor exposure to minimize olfactory stimulation from other females as well as the subjects' own urine and feces. Odor exposure methods were adapted from Schaefer et al. [13]. Female subjects were exposed to either clean air, E female urinary volatiles, or E + P female urinary volatile odors. Subjects were placed in a clean, empty cage $(20 \mathrm{~cm} \times 30 \mathrm{~cm} \times 20 \mathrm{~cm})$ in an odor-free fume hood, and air was passed via odor-free tubing through activated charcoal for purification, followed by drierite, and finally distilled water for rehydration. This purified air was then passed over a $20 \%$ solution of urine in distilled water or over distilled water alone (clean air control). Air flow was adjusted to $1500 \mathrm{ml} / \mathrm{min}$ using a flow meter, and solutions were gently agitated using an orbital shaker during exposure. Urinary volatiles or clean air were blown into the cage via odorless tubing attached to a small funnel affixed to an opening in the side of the cage. Odor exposure sessions began with 40 min of clean air pulsed at 3:1 min on:off intervals, followed by $30 \mathrm{~min}$ of 3:2 min on:off intervals of either clean air, E female urinary volatiles, or $\mathrm{E}+\mathrm{P}$ female urinary volatiles. Ninety minutes after the onset of odor exposure, all subjects were sacrificed via transcardiac perfusion with $0.1 \mathrm{M}$ phosphate buffered saline (PBS) followed by $4 \%$ paraformaldehyde in $0.1 \mathrm{M}$ PBS. All brains were removed and post-fixed in $4 \%$ paraformaldehyde for $2 \mathrm{~h}$, cryoprotected in $30 \%$ sucrose for $48 \mathrm{~h}$, and then were frozen on dry ice. This tissue was subsequently shipped to Boston University on dry ice, whereupon the olfactory bulbs were thawed, blocked and stored in OCT (Tissue-Tek, Elkhart, IN) at $-80^{\circ} \mathrm{C}$. Coronal olfactory bulb sections ( $35 \mu \mathrm{m}$ thickness) were cut on a

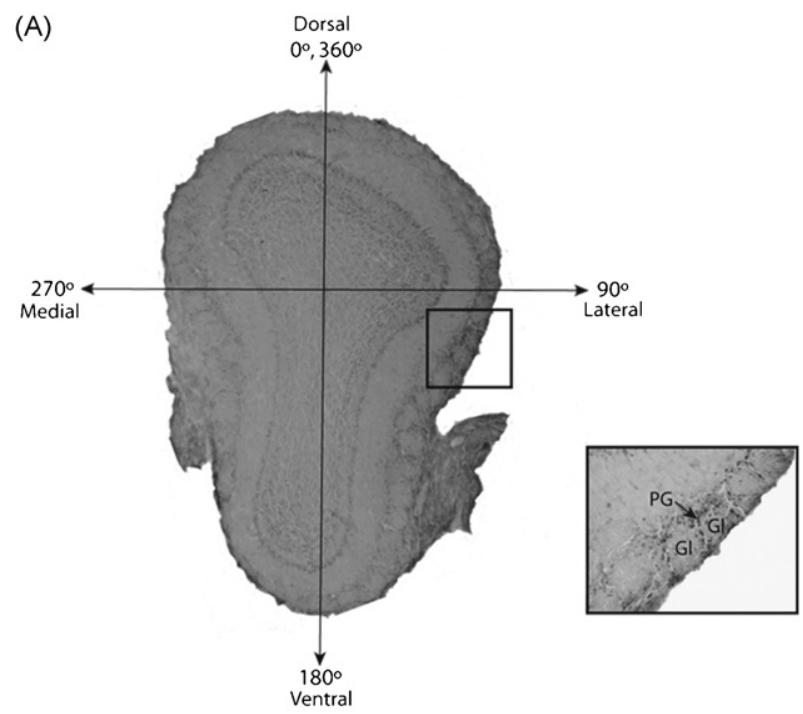

(B)

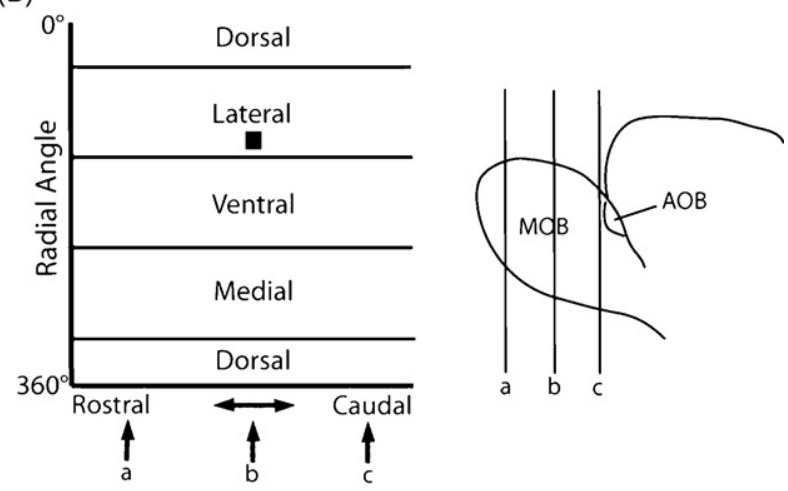

Fig. 1. Identification and mapping of activated glomeruli in the main olfactory bulb in a WT female mouse following exposure to E female volatile urinary odors. (A) An activated glomerulus (Gl) was defined as having two $90^{\circ}$ arcs or a $180^{\circ}$ continuous arc of Fos-IR in the periglomerular cells (PG) surrounding the glomerulus. The representative coronal section shown in panel (A) is located at point (b) in panel (B). A central axis was established $\left(0-180^{\circ}\right)$ using anatomical landmarks, extending from the dorsal mitral cell layer to the ventral mitral cell layer. The lateral, ventral, medial, and dorsal portions of the bulb are located at radial angles of $90^{\circ}, 180^{\circ}, 270^{\circ}$, and $360^{\circ}$, respectively. The location of each activated glomerulus was recorded using the rostral-caudal distance through the bulb as well as the radial angle, and a composite map was formed showing areas of greatest activation throughout the bulb. In panel (B), the location of the slice (b) as well as the activated glomerulus highlighted in the inset are depicted by the black box located in the lateral region of the 2-dimensional map, $\sim 120^{\circ}$ from the central axis. 
cryostat (Leica Microsystems Inc., Bannockburn, IL) at $-18^{\circ} \mathrm{C}$. Free-floating sections were stored in $0.1 \mathrm{M}$ PBS at $4{ }^{\circ} \mathrm{C}$, and every other section was processed for Fos immunocytochemistry (ICC) as previously described [4].

Odor-induced MOB glomerular activation was mapped using Matlab software in conjunction with the GLOM-MAP program [11]. The location of each activated glomerulus was mapped according to radial angle from a central point of origin and rostral-caudal distance through the bulb. Activated glomeruli were defined as having $180^{\circ}$ of continuous Fos activation or two $90^{\circ}$ arcs of Fos activation in the periglomerular cells surrounding the glomerulus $[13,12]$. Olfactory bulbs were sectioned perpendicular to the lateral olfactory tract to insure proper orientation, and sections were analyzed in sequence $(70 \mu \mathrm{m}$ between sections). The rostral and caudal limits for analysis were determined using anatomical landmarks, with the rostral-most section being the first to contain clear mitral cell and external plexiform layers, and the caudal-most section lying just posterior to the accessory olfactory bulb (AOB). Using the thickness of each section $(35 \mu \mathrm{m})$ and its sequence within the sections to be analyzed, we were able to estimate the rostral-caudal location of each activated glomerulus. A central axis $\left(0-180^{\circ}\right)$ extending from the dorsal mitral cell layer to the ventral mitral cell layer was established for each section, and a central point of origin was then determined to be $1 / 3$ the distance from the dorsal portion of the central axis in sections not containing the AOB, and $1 / 3$ the distance from the granular cusp of the AOB in sections containing the AOB. This point of origin was used to determine the radial angle of an activated glomerulus relative to the central axis (Fig. 1).
The rostral-caudal distance and radial angle of each activated glomerulus provided coordinates with which the GLOM-MAP program allowed us to create 2-dimensional color contour plots that show the density of activated glomeruli throughout the MOB. Mapping was accomplished by capturing images of each section using a Nikon digital camera attached to an Olympus microscope. An investigator who was unaware of the treatment group of each subject identified and annotated each activated glomerulus using a microscope and the ACT1 imaging program. Annotated images were then imported into the GLOM-MAP $O B S$ program, in which the central axis was established, and the positions of annotated glomeruli were recorded. Using the statistical toolbox, GLOM-MAP GDB, these data were compiled and smoothed, generating individual contour plots, group averaged contour plots, and statistical comparisons between groups. Mann-Whitney $U$-tests were used to determine significantly different regions of the maps, which are outlined in black [11]. Significant $p$-values were determined using a false discovery rate (FDR) critical value of .025 that was adjusted for multiple Mann-Whitney $U$-test comparisons. The use of an FDR reduced the occurrence of Type I statistical errors [12].

Both WT and ArKO female mice exposed to E urinary volatiles versus $\mathrm{E}+\mathrm{P}$ urinary volatiles showed overlapping but distinguishable patterns of glomerular activation in the MOB (Fig. 2). The regions with the greatest number of activated glomeruli included the rostral-lateral as well as the caudalmedial portions of the MOB. While these hot spots largely overlapped, significant differences between the two groups in patterns of glomerular activation were demonstrated using point

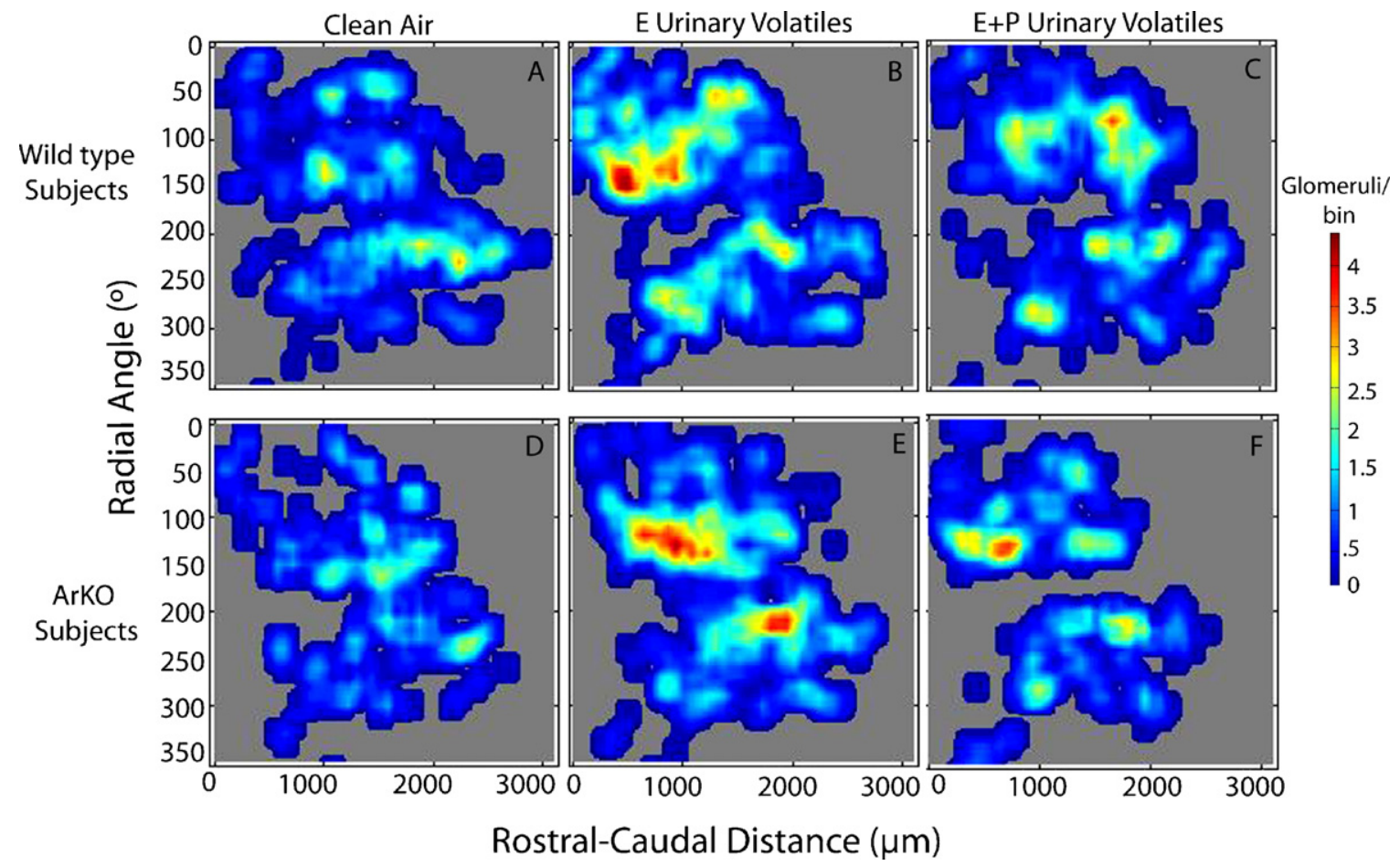

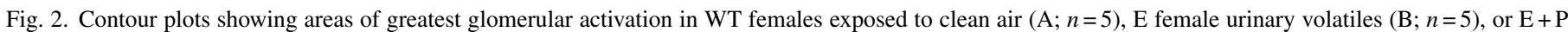

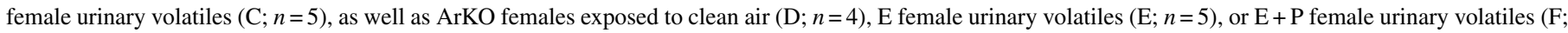
$n=5)$. The scale showing the number of activated glomeruli per bin $\left(72 \mu \mathrm{m}\right.$ thickness, $10^{\circ}$ radial angle $)$ is located to the right of the panels. 


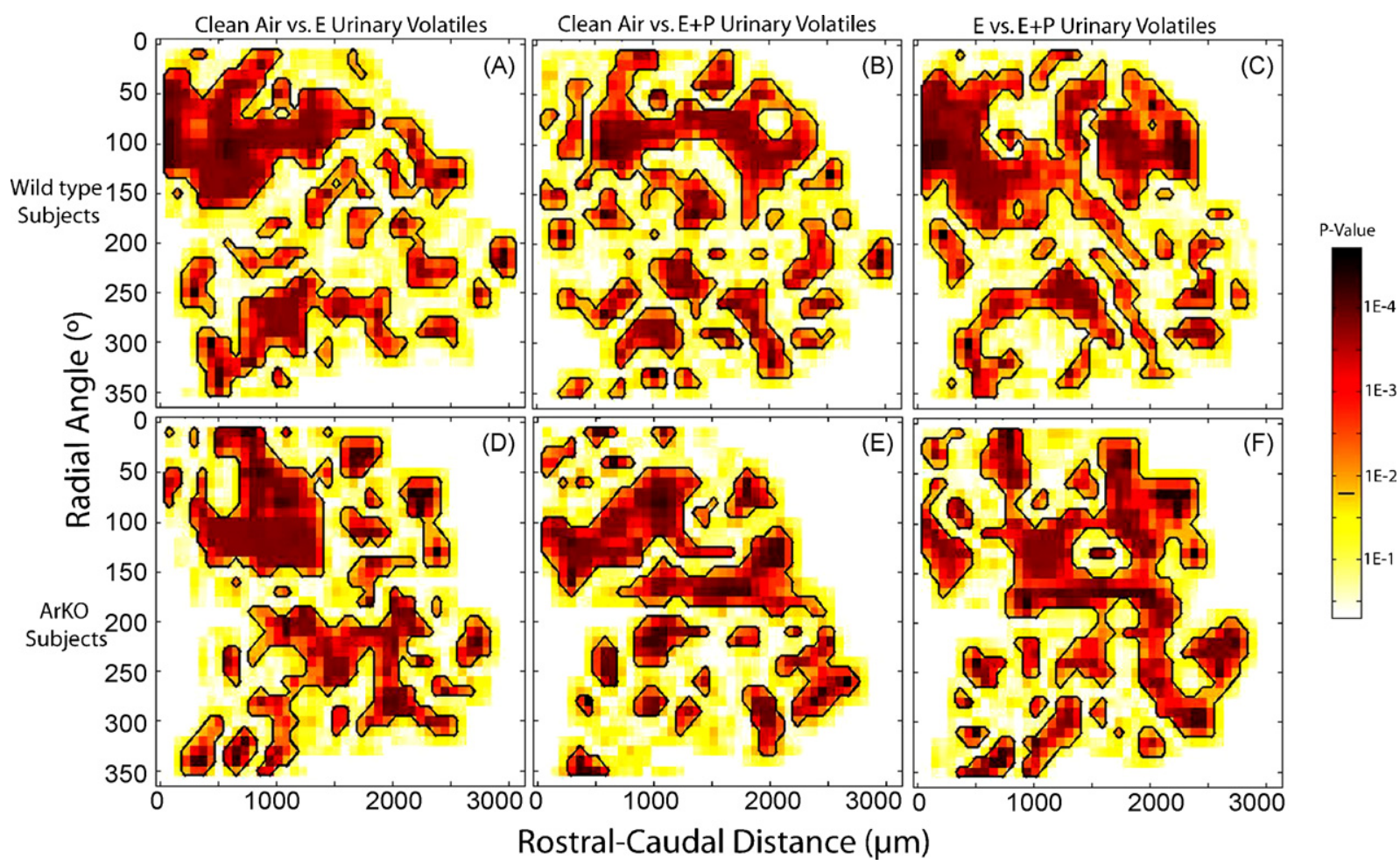

Fig. 3. Regions showing significant differences in glomerular activation in the main olfactory bulb between the contour plots shown in Fig. 2. Panels (A-C) depict statistical comparisons between WT groups, while panels (D-F) show comparisons between ArKO groups. Point by point Mann-Whitney $U$-tests showed widespread significant differences (outlined in black) in all comparisons including clean air vs. E urinary volatiles (A, D), clean air versus $\mathrm{E}+\mathrm{P}$ urinary volatiles (B, E), and $\mathrm{E}$ vs. $\mathrm{E}+\mathrm{P}$ urinary volatiles $(\mathrm{C}, \mathrm{F}) . p<$ false discovery rate (FDR) cutoff value (.025) indicated by the horizontal black bar on the color scale located to the right of the plots.

by point Mann-Whitney $U$-tests (Fig. 3). Significant differences in glomerular activation were observed in WT and ArKO females exposed to clean air versus $\mathrm{E}$ urinary volatiles (Fig. 3A and D) as well as clean air versus $\mathrm{E}+\mathrm{P}$ urinary volatiles (Fig. 3B and $\mathrm{E})$. Finally, similar robust differences in glomerular activation were found in WT as well as ArKO females exposed to $\mathrm{E}$ versus $\mathrm{E}+\mathrm{P}$ urinary volatiles (Fig. $3 \mathrm{C}$ and $\mathrm{F}$ ).

The present data indicate that both WT and ArKO female mice showed distinguishable profiles of MOB glomerular activation in response to urinary volatiles derived from ovariectomized females previously treated with $\mathrm{E}$ as opposed to $\mathrm{E}+\mathrm{P}$. In our previous study [14], ovariectomized, estrogen-treated ArKO females successfully learned to discriminate between urinary volatiles from $\mathrm{E}$ versus $\mathrm{E}+\mathrm{P}$ primed females whereas WT females failed to acquire this task. These behavioral results could possibly result from increased sensitivity to estrogen treatment in the main olfactory pathway of ArKO mice due to perinatal estrogen deprivation. It was previously reported [1] that forebrain estradiol receptor- $\alpha$ expression was upregulated 2-fold in ArKO females. This may, in turn, have upregulated the activational effect of $\mathrm{E}$ in the odor discrimination ability of ArKO females [14]. The present results suggest that the previously observed difference between females of the two genotypes in their ability to discriminate $\mathrm{E}$ vs. $\mathrm{E}+\mathrm{P}$ urinary volatiles [14] cannot be attributed to the absence of differential MOB profiles of glomerular activation in WT females following exposure to the two different types of urinary volatiles. Apparently, the fail- ure of WT females to discriminate behaviorally between $\mathrm{E}$ and $\mathrm{E}+\mathrm{P}$ urinary volatiles reflected a deficit in the processing of odor inputs by more central segments of the olfactory projection pathway. Conversely, the augmented capacity of ArKO females to discriminate between these two types of female urinary odors [14] probably reflected a facilitation in the central processing of these odor cues resulting from perinatal exposure to estradiol as opposed to an effect on the initial processing of these cues by glomeruli in the MOB.

\section{Acknowledgements}

This work was supported by NICHD grant HD044897. J.Bakker is a Research Associate at the Fonds National de la Recherche Scientifique (Belgium). We thank Ernesto Salcedo for his help with the glomerular analysis software.

\section{References}

[1] V.R. Agarwal, C.M. Sinton, C. Liang, C. Fisher, D.C. German, E.R. Simpson, Upregulation of estrogen receptors in the forebrain of aromatase knockout (ArKO) mice, Mol. Cell. Endocrinol. 162 (2000) 9-16.

[2] J. Bakker, S. Honda, N. Harada, J. Balthazart, Sexual partner preference requires a functional aromatase (Cyp19) gene in male mice, Horm. Behav. 42 (2002) 158-171.

[3] N. Bodyak, B. Slotnick, Performance of mice in an automated olfactometer: odor detection, discrimination and odor memory, Chem. Senses 24 (1999) $637-645$. 
[4] H.A. Halem, J.A. Cherry, M.J. Baum, Vomeronasal neuroepithelium and forebrain Fos responses to male pheromones in male and female mice, J. Neurobiol. 39 (1999) 249-263.

[5] S. Honda, N. Harada, S. Ito, Y. Takagi, S. Maeda, Disruption of sexual behavior in male aromatase-deficient mice lacking exons 1 and 2 of the cyp19 gene, Biochem. Biophys. Res. Commun. 252 (1998) 445449.

[6] S.D. Liberles, L.B. Buck, A second class of chemosensory receptors in the olfactory epithelium, Nature 442 (2006) 645-650.

[7] W. Lin, J. Arellano, B. Slotnick, D. Restrepo, Odors detected by mice deficient in cyclic nucleotide-gated channel subunit A2 stimulate the main olfactory system, J. Neurosci. 24 (2004) 3703-3710.

[8] D.Y. Lin, S.Z. Zhang, E. Block, L.C. Katz, Encoding social signals in the mouse main olfactory bulb, Nature 434 (2005) 470-477.

[9] K.L. Martel, M.J. Baum, Sexually dimorphic activation of the accessory olfactory bulb in mice by urinary volatiles, Eur. J. Neurosci. (2007), in press.
[10] K.J. Ressler, S.L. Sullivan, L.B. Buck, A molecular dissection of spatial patterning in the olfactory system, Curr. Opin. Neurobiol. 4 (1994) 588-596.

[11] E. Salcedo, C. Zhang, E. Kronberg, D. Restrepo, Analysis of traininginduced changes in ethyl acetate odor maps using a new computational tool to map the glomerular layer of the olfactory bulb, Chem. Senses 30 (2005) 615-626.

[12] M.L. Schaefer, K. Yamazaki, K. Osada, D. Restrepo, G.K. Beauchamp, Olfactory fingerprints for major histocompatibility complex-determined body odors II: relationship among odor maps, genetics, odor composition, and behavior, J. Neurosci. 22 (2002) 9513-9521.

[13] M.L. Schaefer, D.A. Young, D. Restrepo, Olfactory fingerprints for major histocompatibility complex-determined body odors, J. Neurosci. 21 (2001) 2481-2487.

[14] D.W. Wesson, M. Keller, Q. Douhard, M.J. Baum, J. Bakker, Enhanced urinary odor discrimination in female aromatase knockout mice, Horm. Behav. 49 (2006) 580-586. 